\title{
PROJETOS PEDAGÓGICOS DE CURSOS DE SAÚDE E SUA ARTICULAÇÃO COM AS POLÍTICAS PÚBLICAS PARA A FORMAÇÃO EM SAÚDE NO BRASIL
}

Lucimar D. S. Salvador ${ }^{1}$

Paulo A. Sant'Anna ${ }^{1}$

\section{RESUMO}

Este estudo objetivou compreender como os projetos pedagógicos dos cursos de saúde de uma instituição pública de ensino superior articulam, nas dimensões política e pedagógica, as orientações curriculares preconizadas nas Diretrizes Curriculares Nacionais - DCNs (2001, 2002) e no Programa Nacional de Reorientação da Formação Profissional em Saúde - PróSaúde (2005). Trata-se de um estudo de caso, exploratório e de enfoque qualitativo, com uso da técnica da pesquisa documental e submissão dos dados à análise de conteúdo. Os resultados revelaram que os Projetos Pedagógicos de Cursos (PPCs) articulam, na dimensão política, um ideário de formação coerente com as DCNs e o Pró-Saúde. Entretanto, na dimensão pedagógica, não alcançaram o grau proposto nessas políticas, devido à predominância de currículos disciplinares com dissociação entre os ciclos básico e profissional. Isso sinaliza a necessidade de investimento institucional na capacitação e no desenvolvimento docente para as mudanças propostas. A integração ensino/trabalho exige esforço político-gerencial para a transformação dos cenários de práticas da rede pública de serviços em campos de formação profissional.

Palavras-chave: Formação em saúde. Diretrizes Curriculares. Pró-Saúde. Currículo.

\footnotetext{
${ }^{1}$ Universidade Federal dos Vales do Jequitinhonha e Mucuri, Diamantina, MG, Brasil.
} 


\section{PEDAGOGICAL PROJECTS OF HEALTH COURSES AND THEIR ARTICULATION WITH THE PUBLIC POLICIES FOR HEALTH TRAINING IN BRAZIL}

Lucimar D. S. Salvador

Paulo A. Sant'Anna

\section{ABSTRACT}

This study aimed to understand how the pedagogical projects of the health courses of a public institution of higher education articulate, in the political and pedagogical dimensions, the curricular guidelines recommended in the National Curriculum Guidelines (Diretrizes Curriculares Nacionais - DCNs, 2001, 2002) and in the National Program for the Reorientation of Health Professional Training - Pró-Saúde (2005). It is an exploratory case study, with a qualitative approach, using the documentary research technique and submission of data to content analysis. The results revealed that the Pedagogical Projects of Courses (PPCs) articulate, in the political dimension, an ideology of formation coherent with the DCNs and the Pró-Saúde. However, in the pedagogical dimension, they did not reach the proposed level in these policies, due to the predominance of disciplinary curricula with dissociation between the basic and the professional cycles. This reveals the need for institutional investments in teacher's training and development for the proposed changes. The teaching/working integration requires political-managerial efforts in order to transform the public service practice scenarios into professional training fields.

Keywords: Health training. Curricular Guidelines. Pró-Saúde. Curriculum. 


\section{INTRODUÇÃO}

A formação superior em saúde no Brasil vem sendo amplamente discutida e reformulada nas duas últimas décadas, visando à transformação das práticas profissionais e da própria organização do trabalho, com o objetivo de atender às necessidades de saúde da população e contribuir para a consolidação do Sistema Único de Saúde (SUS) (BRASIL, 2007).

Nesse contexto, diversas políticas públicas foram implementadas pelo governo brasileiro, objetivando produzir mudanças no ensino de graduação. As Diretrizes Curriculares Nacionais (DCNs), publicadas pelo Ministério da Educação (MEC), a partir de 2001, orientam a respeito da construção de Projetos Pedagógicos de Cursos (PPCs) e currículos flexíveis e inovadores, com potencial para reorientar a formação no sentido de construir um perfil acadêmico e profissional com competências e habilidades para atuar na resolução dos problemas de saúde dos indivíduos e das coletividades (BRASIL, 2001a, 2001b, 2002a, 2002b, 2002c).

As DCNs visam assegurar a aprendizagem centrada no estudante; a construção de um currículo integrado, destacando-se a importância da articulação do ensino com os serviços de saúde e da integração teoria/prática vinculada a esse contexto; a utilização de novas metodologias de ensino; e a concepção de saúde associada às condições de vida (LOPES NETO et al., 2007).

O projeto pedagógico, documento norteador das ações de um curso, expressa um compromisso com a formação profissional, portanto, como afirma Veiga (2000, p. 13), “é uma ação intencional com sentido explícito, com um compromisso definido coletivamente". $\mathrm{Na}$ dimensão política, o PPC assume o compromisso com a formação de um cidadão para um tipo de sociedade que conduz à transformação social ou reafirma as desigualdades existentes. Se a intencionalidade reflete propósitos da transformação, o projeto se compromete com a formação de sujeitos críticos, participativos, éticos, compromissados em efetivar mudanças no contexto social. Essa intencionalidade de formação se efetiva na dimensão pedagógica, por meio do currículo, definindo e realizando um conjunto de ações educativas necessárias ao cumprimento desse propósito. Dessa forma, deve ser tomado como estratégico no sentido de promover mudanças nas condições presentes na formação (GATTÁS, 2006).

Conforme Davini (1994), a opção por um currículo integrado na área da saúde possibilita a articulação dinâmica entre teoria e prática, trabalho e ensino, ensino e comunidade, 
favorecendo relações entre os problemas e as hipóteses de solução para eles, considerando as características socioculturais do meio em que esse processo se desenvolve.

Essa proposta de currículo permite integrar os conhecimentos dos ciclos básico e profissional, na perspectiva da interdisciplinaridade, que, para Silva et al. (2008, p. 19), significa "incluir o processo de síntese (integração) propondo uma abordagem integral das pessoas, sociedade e meio ambiente, assim como de seus problemas e necessidades". Nessa perspectiva, são construídos eixos integradores, que passam a articular o conhecimento das disciplinas em torno dos processos que se quer analisar, partindo-se de problemas para a construção da aprendizagem significativa e da formação orientada por competência, promovendo a inserção de professores e estudantes em cenários reais da prática profissional.

Estudos realizados em 2005, sobre a implantação das DCNs nos cursos de Enfermagem, Medicina e Odontologia, verificaram que essas políticas, por si só, não conseguiram promover as mudanças necessárias nos projetos pedagógicos e na formação dos profissionais de saúde. Como características recorrentes nos cursos, ainda persistiam fragilidades, tais como "currículo constituído de disciplinas isoladas, ainda baseado no currículo mínimo, a existência de ciclo básico, pré-clínico e clínico e a ausência de integração com a rede de serviços" (BRASIL, 2006, p. 155).

Nesse contexto, foi criado, em 2005, o Programa Nacional de Reorientação da Formação Profissional em Saúde (Pró-Saúde), como política indutora das mudanças propostas pelas DCNs e com o objetivo de incentivar transformações no processo de formação profissional, considerando as interfaces entre ensino, saúde e trabalho, enfocando três eixos: orientação teórica, cenários de prática e orientação pedagógica (BRASIL, 2007).

Considerando a adesão dos cursos de graduação em saúde de uma instituição federal de ensino superior (IFES) ao Pró-Saúde, este estudo buscou responder às seguintes indagações: como os projetos pedagógicos articulam as orientações das DCNs e do Pró-Saúde na organização das dimensões política e pedagógica? Ocorreram mudanças na perspectiva da integração curricular e do uso de metodologias ativas de ensino/aprendizagem?

O estudo apresentou como objetivo compreender a inserção das orientações das Diretrizes Curriculares Nacionais e do Pró-Saúde na organização das dimensões política e pedagógica 
dos projetos pedagógicos dos cursos de graduação em saúde em Enfermagem, Farmácia, Fisioterapia, Nutrição e Odontologia dessa IFES.

\section{METODOLOGIA}

Tratou-se de um estudo exploratório de enfoque qualitativo, adotando o delineamento de estudo de caso. De acordo com Triviños (1987, p. 133), o estudo de caso "é uma categoria de pesquisa cujo objeto é uma unidade que se analisa aprofundadamente". Essa unidade deve figurar como parte relevante de um todo, isto é, ser significativa e, por isso, permitir fundamentar um julgamento ou propor uma intervenção.

A pesquisa envolveu análise documental das fontes primárias: projetos Pró-Saúde (2005, 2007), PPCs de Enfermagem, Farmácia, Fisioterapia, Nutrição e Odontologia $(2008,2009)$ e planos de ensino das disciplinas da área profissional, ministradas no primeiro e no segundo semestres de 2013. As fontes de análise foram obtidas por meio de cartas de anuência da Pró-Reitoria de Graduação e da direção da Faculdade de Ciências Biológicas e da Saúde da instituição.

Para análise dos dados, adotou-se o procedimento de análise de conteúdo (BARDIN, 2011). A partir da leitura minuciosa das políticas públicas para a formação em saúde, com destaque para as DCNs e o Pró-Saúde, bem como da literatura sobre as temáticas - projeto pedagógico, currículo e formação -, emergiram categorias e subcategorias a partir de duas dimensões do currículo: dimensão política e dimensão pedagógica. Para a primeira, delimitou-se a categoria concepção de saúde (C.1) e duas subcategorias: formação profissional (C.1.1) e atuação profissional (C.1.2). Para a segunda dimensão, delimitou-se a categoria concepção de currículo (C.2) e cinco subcategorias: integração ciclo básico/ciclo profissional (C.2.1), integração ensino/trabalho (C.2.2), interdisciplinaridade (C.2.3), flexibilidade (C.2.4) e metodologias ativas (C.2.5). O roteiro de análise foi construído e, posteriormente, validado por juízes, a partir da definição conceitual de cada categoria e subcategoria, com base nas DCNs e no PróSaúde, documentos que subsidiaram a análise dos projetos pedagógicos e a identificação dos indicadores relacionados.

$\mathrm{Na}$ análise das informações, compararam-se os indicadores extraídos dos PPCs, tendo por base o roteiro de análise. Assim, buscou-se analisar na "C.1" a concepção de saúde presente nesses PPCs, sob o enfoque do modelo ampliado, bem como a coerência e a articulação dessa 
concepção com as subcategorias "C.1.1" formação profissional e "C.1.2" atuação profissional, focalizando-se também os referenciais propostos pelas DCNs e pelo Pró-Saúde. Na "C.2", analisou-se a concepção de currículo presente nos PPCs, sob a ótica da integração curricular, buscando-se evidenciar nas subcategorias "C.2.1" integração ciclo básico/profissional, "C.2.2" integração ensino/trabalho, "C.2.3" interdisciplinaridade, "C.2.4" flexibilidade e "C.2.5" metodologias ativas, elementos que indicassem a materialização das proposições pedagógicas/ curriculares estabelecidas pelas políticas públicas para a formação em saúde.

$\mathrm{Na}$ apresentação dos resultados e na discussão, nomearam-se os cursos pesquisados com os códigos: $A, B, C, D$ e E, a fim de preservar o anonimato, conforme os preceitos éticos da pesquisa.

\section{RESULTADOS E DISCUSSÃO}

\section{Dimensão Política}

Concepção de Saúde (C.1)

Na dimensão política, os projetos pedagógicos dos cinco cursos da saúde analisados indicam que a concepção de saúde subjacente à formação profissional fundamenta-se no "conceito ampliado" (BUSS, 1999), reconhecendo-a como resultante de determinantes sociais, associada a um amplo espectro de fatores relacionados com a qualidade de vida (UFVJM, 2008a, 2008b, 2008c, 2009a, 2009b).

Essa concepção presente nos PPCs traz implícita a ideia de uma abordagem mais ampla da saúde, podendo sinalizar a compreensão dos cursos acerca da necessidade de se considerarem os aspectos históricos, econômicos, sociais, culturais, biológicos, ambientais e psicológicos que configuram uma determinada realidade, ao tratar o processo saúde/doença.

A compreensão dos conceitos de saúde/doença, segundo Batistella $(2007$, p. 27), possibilita "rediscutir as intervenções sobre esta mesma realidade, traduzindo-se nas opções de conhecimento necessário, no desenvolvimento de métodos, técnicas e instrumentos para a intervenção, de modo a promover a saúde e evitar a doença". 
Formação Profissional (C.1.1)

Na subcategoria formação profissional, os PPCs trazem uma concepção condizente com as DCNs e o Pró-Saúde, isto é, a de um profissional generalista, humanizado, crítico e reflexivo, capaz de desenvolver a atenção integral, pautada pela compreensão da realidade social, cultural e econômica e das necessidades sociais de saúde, tanto em nível individual quanto no coletivo. Esse profissional deverá desenvolver ações de prevenção, promoção, proteção e reabilitação da saúde, com responsabilidade social e compromisso com a cidadania, adquirindo um conjunto de competências e habilidades gerais e específicas, relacionadas à atenção à saúde, à tomada de decisões, à comunicação, à liderança, à administração/gerenciamento e à educação permanente (UFVJM, 2008a, 2008b, 2008c, 2009a, 2009b).

A concepção de formação apontada nos PPCs explicita a intencionalidade de uma educação cidadã coerente com a educação problematizadora defendida por Freire (2013, p. 100), na qual "os educandos em suas relações com o mundo vão desenvolvendo o seu poder de captálo e de compreendê-lo, estabelecendo uma forma autêntica de pensar e atuar criticamente, no sentido de transformar a realidade".

\section{Atuação Profissional (C.1.2)}

Nessa subcategoria, os PPCs indicam a atuação dos futuros profissionais nos diferentes níveis de atenção à saúde e em diferentes cenários, incluindo-se equipamentos sociais (creches, escolas, instituições para idosos), serviços públicos (Estratégia de Saúde da Família - ESF, Unidades Básicas de Saúde - UBS, ambulatórios, hospitais) e privados de saúde (UFVJM, 2008a, 2008b, 2008c, 2009a, 2009b).

Portanto, na dimensão política, depreende-se que os PPCs dos cursos da saúde analisados articulam intencionalidades expressas na concepção de saúde, de formação e de atuação profissional coerentes com as proposições das DCNs e do Pró-Saúde. Isso sinaliza a compreensão dos cursos acerca dos paradigmas atuais da formação em saúde, que, de acordo com Macedo et al. (2011, p. 230), possibilitam "definir as decisões e escolhas que irão determinar certas configurações curriculares e a formação profissional". 


\section{Dimensão Pedagógica}

\section{Concepção de Currículo (C.2)}

Na dimensão pedagógica, em que reside a possibilidade de efetivação da intencionalidade da instituição, que é a formação do cidadão (VEIGA, 2000), emergem, nos PPCs dos cinco cursos analisados, diversas concepções de currículo, tais como currículo integrado e orientado por competência e currículo pautado na interdisciplinaridade. Essas concepções indicam a percepção dos agentes formadores sobre a pertinência de integração de áreas do conhecimento no processo formativo, de forma a conduzir ao desenvolvimento das competências e habilidades definidas para a construção do perfil profissional (UFVJM, 2008a, 2008b, 2008c, 2009a, 2009b).

\section{Integração Ciclo Básico e Profissional (C.2.1)}

No campo do currículo, verifica-se que apenas o PPC " $A$ " indica avanços na perspectiva da integração de conteúdos da área profissional, organizados em macrodisciplinas (UFVJM, 2009b). Assim, nos PPCs "B", "C", "D" e "E", embora se observem mudanças curriculares no sentido da inserção de disciplinas da área profissional nos períodos iniciais, da criação de disciplinas da saúde coletiva e da saúde pública, da distribuição dos estágios curriculares ao longo dos períodos (UFVJM, 2008a, 2008b, 2008c, 2009a), a tendência predominante na organização curricular prescrita é de uma estrutura disciplinar, com dissociação entre os ciclos básico e profissionalizante, sem evidências de integração vertical e horizontal, características do currículo formal/tradicional (DAVINI, 1994).

Essa forma de organização, segundo Bordas (2007, p. 86), “[...] é um poderoso entrave ao desenvolvimento de uma visão integrada do conhecimento e dificulta a compreensão da relação teoria-prática, essencial ao processo de construção do conhecimento [...]". Além disso, por impossibilitar o exercício da conexão entre os conhecimentos e a contextualização deles, pode induzir a uma formação reducionista, recortada e procedimento-centrada, impactando no perfil do profissional a ser formado (ALBUQUERQUE et al., 2009). 
A integração ensino/trabalho, segundo Lopes Neto et al. (2007, p. 633), é "essencial para superar a formação centrada no conhecimento fisiopatológico e desagregada da realidade sócio-sanitária e da prestação do cuidado na rede SUS". Os PPCS " $A$ ", "B", "D" e "E" indicam essa prática fundamental e, embora ao longo do processo de formação busquem inserir os estudantes em cenários de práticas diversificados, por meio das disciplinas curriculares e dos estágios supervisionados (UFVJM, 2008a, 2008b, 2008c, 2009a, 2009b), revela-se ainda, na maioria dos cursos, uma tendência à inserção tardia dos estudantes nos serviços públicos de saúde, em especial na atenção básica, contrariamente à proposição das DCNs e do PróSaúde. Revela-se também a concentração de atividades curriculares práticas em laboratórios e clínicas internas à instituição, fatores que podem sinalizar o distanciamento e o descompasso ainda existentes entre a formação em saúde e as necessidades de saúde da população.

Esses fatores podem estar associados à carência de condições estruturais da rede SUS do município onde estão inseridos os cursos de graduação da saúde e em municípios circunvizinhos. Reconhece-se a emergência de ações intersetoriais para se enfrentar o desafio da necessária articulação ensino/trabalho, viabilizando espaços de ensino/aprendizagem que possibilitem aos estudantes, conforme aponta Macedo et al. (2011, p. 230), "reflexões críticas e propositivas sobre os processos de trabalho em saúde, sobre as relações ainda verticalizadas entre os profissionais e os usuários e sobre a hierarquização entre os saberes profissionais".

Para que a integração ensino/trabalho se efetive na atenção primária e em outros níveis do sistema, exige-se um esforço político e gerencial da gestão das instituições de ensino e serviços de saúde, no sentido de ampliar os cenários de prática e preparar a rede de saúde, visando transformá-la em campo de formação (SOUZA; ZEFERINO; DA ROS, 2011).

\section{Interdisciplinaridade (C.2.3)}

Na subcategoria interdisciplinaridade, verificou-se que essa concepção permeia fortemente a maioria dos PPCs da área da saúde da Instituição de Educação Superior (IES). Com relação às proposições de práticas interdisciplinares, indica-se no PPC " $A$ " a possibilidade da integração de disciplinas afins, viabilizada pelas macrodisciplinas na organização curricular e no processo de ensino/aprendizagem; no PPC " $B$ " indica-se a interação entre as disciplinas nas ações de promoção, prevenção, recuperação e reabilitação da saúde; e no PPC "E" aponta- 
se a possibilidade de realização de estudos e atividades interdisciplinares que integrem os docentes de diferentes áreas, bem como a adoção de estratégias avaliativas. O PPC "C" não apresenta informações sobre a interdisciplinaridade, e, no PPC " $D$ ", acena-se para a integração de conteúdos na organização curricular, a qual não se configura no currículo prescrito (UFVJM, 2008a, 2008b, 2008c, 2009a, 2009b).

As proposições de práticas interdisciplinares apresentadas nos PPCS "A", "B" e "E" não permitem clareza quanto à articulação entre os componentes biológicos, clínicos e sociais no processo de ensino/aprendizagem, tampouco em que níveis essas interações se efetivam. No entanto, podem sinalizar possibilidades de rupturas com as práticas tradicionais, aproximandose do referencial de "atitude" apresentado por Fazenda (1996), de busca de alternativas para conhecer mais e melhor, de envolvimento, compromisso e reciprocidade diante do conhecimento.

De um modo geral, pode-se depreender que ainda é incipiente, no âmbito dos PPCs, a orientação das práticas pedagógicas pelo enfoque integrado/interdisciplinar, no sentido proposto pelas DCNs e pelo Pró-Saúde, constituindo um desafio a ser superado, o que exige atitude, abertura e engajamento dos educadores para a efetivação dessa prática. 0 trabalho interdisciplinar requer "romper com hábitos e acomodações para buscar algo novo e desconhecido, desmantelar as fronteiras artificiais do conhecimento para propiciar uma visão interdisciplinar" (THIESEN, 2007, p. 96), favorecendo, assim, o enfrentamento de problemas complexos da realidade de saúde e propiciando a formação integral dos discentes para exercerem criticamente a cidadania.

Nessa direção, Bordas (2007) propõe como ações necessárias nos âmbitos institucional, departamental e de cursos: a promoção de discussões teóricas coletivas sobre a interdisciplinaridade, o que possibilitará pensar e desenhar projetos inovadores de ensino, para lidar com a complexidade da formação profissional; e a oferta de espaços institucionais para a proposição de projetos interdisciplinares, que articulem professores e pesquisadores em torno de temas e problemas que precisam ser tratados sob múltiplos olhares, garantindo condições para que sejam efetivados. 
Flexibilização (C.2.4)

No que concerne à subcategoria flexibilização, indica-se, nos PPCs dos cinco cursos da área da saúde, a reserva de carga horária curricular para o desenvolvimento de atividades complementares, incluindo, dentre outras, pesquisa, ensino, extensão, participação em evento científico, estágios extracurriculares, monitorias, cursos de língua estrangeira, disciplinas eletivas (UFVJM, 2008a, 2008b, 2008c, 2009a, 2009b).

Esse aspecto, consoante com as orientações das DCNs, assinala a abertura dos currículos dos cursos da área da saúde para uma formação mais abrangente em termos acadêmicos, profissionais e culturais, possibilitando ao estudante graus de liberdade, bem como autonomia para complementar a formação.

\section{Metodologias (C.2.5)}

Quanto às metodologias, apenas o PPC "C" não apresenta elementos que indiquem a concepção adotada pelo curso. Os PPCs "A", "B", "D" e "E" evidenciam, nas concepções propostas, elementos que caracterizam as metodologias ativas, indicando: aprendizagem centrada no discente como sujeito e mediada pelo professor como facilitador desse processo; oferta de condições de análise e reflexão crítica dos problemas de saúde/doença e das reais necessidades de saúde da população; estímulo à reflexão sobre a prática profissional, à busca de informações e à teorização; discussão em grupos, favorecendo a apropriação e a produção dos conhecimentos (UFVJM, 2008a, 2008b, 2008c, 2009a, 2009b).

Os planos de ensino das disciplinas da área profissional dos cinco cursos da área da saúde, ministradas no 1 으 e 20 semestres de 2013, indicaram o estudo de casos reais ou simulados como a metodologia mais comum utilizada pelos cursos. Essa metodologia também potencializa e induz a participação ativa do discente na construção do conhecimento, pois possibilita a análise de situações encontradas no cotidiano da profissão, em diferentes ângulos, contribuindo para a tomada de decisão (BERBEL, 2011; GIL, 2011). Contudo, os planos de ensino analisados indicam também a adoção de uma diversidade de métodos e técnicas para desenvolver as atividades curriculares, envolvendo, dentre outros, aulas teóricas e práticas de laboratório, exposição dialogada, seminários, observação da prática clínica em grupos, leitura e discussão de artigos científicos. Essas informações sugerem que os currículos dos cursos da área da saúde não privilegiam, ainda, um ensino pautado na adoção de metodologias ativas, ancoradas na 
problematização como método predominante e orientador do processo de aprendizagem, centradas no estudante e com atividades estruturadas a partir das necessidades de saúde da população (BRASIL, 2007).

As informações extraídas dos PPCs indicam, na dimensão política, a articulação com as orientações das DCNs e do Pró-Saúde, expressa na concepção ampliada de saúde e na intencionalidade de formação e atuação do profissional, a qual se coaduna com os pressupostos de uma formação cidadã, capaz de propiciar o conhecimento da realidade de saúde, bem como a intervenção no sentido de transformá-la.

Na dimensão pedagógica, a predominância de currículos disciplinares, a dissociação entre os ciclos básico e profissional, a aproximação tardia dos estudantes com os serviços públicos de saúde, a incipiência das proposições interdisciplinares e a necessidade de utilizar a problematização como método orientador do processo de ensino/aprendizagem são fatores presentes na maioria dos PPCs, os quais sinalizam que não houve, no conjunto dos cursos, mudanças curriculares e de modelo pedagógico no grau proposto pelas DCNs e pelo Pró-Saúde. Isso pode revelar, dentre outros fatores, a não compreensão dos princípios epistemológicos e pedagógicos expressos nas DCNs e no Pró-Saúde; a ausência de preparação pedagógica do corpo docente para a implantação de mudanças curriculares inovadoras; e a dificuldade de diálogo entre as áreas de conhecimento, devido à estrutura departamental que sustenta a organização dos cursos.

As dificuldades para se promoverem as reformas curriculares propostas pelo Pró-Saúde também foram constatadas em avaliação do programa pelo Ministério da Saúde (MS), sendo apontados como fatores limitadores: a resistência dos docentes em mudar as práticas pedagógicas devido à falta de capacitação deles, dos discentes e familiares para tal mudança; a incoerência entre os modelos de gestão das IES, centralizados na organização departamental, dificultando a implantação de um currículo mais integrado (BRASIL, 2009).

Para Gordan (2004), esses fatores precisam ser levados em consideração no planejamento de mudanças curriculares inovadoras, visando aumentar as possibilidades de inserção docente na nova situação. Nessa perspectiva, reforça-se a necessidade de se garantir, em âmbito institucional, processo de desenvolvimento docente que atue como um pilar estruturador para as mudanças pretendidas no campo da formação profissional em saúde, pois, somente pela 
compreensão dos propósitos de mudança, será possível o engajamento e comprometimento dos envolvidos com os resultados.

É importante mencionar que este estudo analisou os PPCs prescritos para os cursos de graduação da área da saúde da IFES, o que nos permite clareza quanto à possibilidade de haver diferença entre os currículos prescritos e os que realmente estão em ação. É preciso considerar, também, as limitações do estudo no que concerne às atividades de pesquisa e extensão, as quais, apesar de não atingirem o contingente de estudantes de um curso, contribuem para o alcance da formação profissional idealizada nos PPCs.

\section{CONSIDERAÇÕES FINAIS}

O presente estudo revelou que os PPCs prescritos para os cinco cursos da área da saúde da IES articulam, na dimensão política, intencionalidades expressas na concepção de saúde, de formação e atuação profissional coerentes com as orientações preconizadas pelas DCNs e pelo Pró-Saúde. Portanto, esses documentos apresentam um ideário filosófico e político condizente com os pressupostos de uma formação cidadã, que se traduz no perfil de um profissional generalista, humanizado, crítico e reflexivo, capaz de conhecer, compreender e atuar na realidade de saúde, no sentido de transformá-la.

No entanto, o alcance da formação desse perfil profissional, voltado para a oferta de uma assistência integral à saúde, mais contextualizada e resolutiva, requer ainda mudanças curriculares e no processo pedagógico, possibilitando também a aproximação com os serviços para o reconhecimento do perfil epidemiológico e das necessidades de saúde da população, em especial a local e a regional.

Nessa perspectiva, apontam-se ainda como necessários na dimensão pedagógica dos PPCs da maioria dos cursos da área da saúde elementos que merecem reflexão por parte da instituição: o redirecionamento curricular na perspectiva da integração dos conhecimentos das disciplinas; a inserção precoce dos estudantes na rede pública de saúde, propiciando a vivência da teoria/prática num contexto profissional real; o estímulo ao exercício da interdisciplinaridade, possibilitando a compreensão dos problemas de saúde em múltiplas dimensões; e a priorização do uso de metodologias ativas, propiciando a participação ativa e crítica do estudante na construção do próprio conhecimento. 
Com base nas reflexões realizadas, pode-se depreender a necessidade do desenvolvimento de estratégias que potencializem mudanças nos elementos propostos na dimensão pedagógica dos PPCs, favorecendo práticas curriculares que possibilitem concretizar o ideário de formação expresso na dimensão política, em consonância com os pressupostos epistemológicos e metodológicos preconizados pelas DCNs e pelo Pró-Saúde.

As mudanças curriculares e as novas concepções de ensino/aprendizagem, apesar de serem temas amplamente discutidos em diversas áreas do conhecimento, são complexas e lentas. Entende-se que dependem, em grande medida, da compreensão, pelos docentes, do processo de mudanças na formação superior em saúde no Brasil, dos fundamentos teóricos, filosóficos e políticos da área e da aplicação desses à realidade prática, de modo a possibilitar uma reflexão problematizadora sobre as próprias práticas pedagógicas. A tomada de consciência sobre essas questões permitirá o posicionamento ético/político e a definição dos rumos a se seguir na elaboração dos PPCs, norteando a construção de currículos que considerem as necessidades da sociedade e da formação do perfil profissional.

Para tanto, colocam-se grandes desafios à instituição no exercício da autonomia e da responsabilidade social, os quais exigem direcionamento e esforço conjunto das gestões da Pró-Reitoria de Graduação, dos cursos e departamentos. O primeiro desafio diz respeito ao investimento e ao fortalecimento das políticas de formação e desenvolvimento docente, focalizando o diálogo entre os cursos e departamentos, ampliando os espaços de debates e reflexões sobre as intencionalidades e as práticas curriculares que permeiam os processos formativos na instituição, mediatizados pelos contextos histórico, político e social que envolvem o cenário da saúde no Brasil e pelas políticas da instituição e públicas nacionais para a formação profissional.

O segundo desafio diz respeito a uma maior aproximação/articulação entre a gestão da IES e a gestão do sistema público de saúde, visando fortalecer parcerias para adequação dos cenários de práticas desses serviços e a transformação desses em campos de formação. Desse modo, a formação dos profissionais de saúde poderá ser fortalecida nesses espaços, que constituem os diferentes cenários da vida real e da produção do cuidado em saúde.

Tomar o projeto pedagógico como instrumento estratégico de gestão e mudanças na formação consiste em um terceiro desafio, que requer comprometimento dos gestores e formadores com as ações propostas. Para tanto, são fundamentais a socialização do PPC com todos os 
envolvidos no processo formativo (docentes, discentes, demais servidores e comunidade externa); a avaliação contínua da implantação do PPC e dos resultados alcançados; a coerência das proposições, em termos de formação, com as práticas desenvolvidas, de modo a possibilitar a identificação das necessidades de reestruturação.

Longe de apontar caminhos, compreende-se que essas proposições possam contribuir para a superação das fragilidades apontadas nos PPCs. Embora constituam verdadeiros desafios, por incidirem em campos de interesses e poder, instaurando tensões e conflitos, acredita-se que são necessárias e indispensáveis para que se promovam rupturas com o instituído e se instalem mudanças nas práticas formadoras dos profissionais da saúde.

\section{REFERÊNCIAS}

ALBUQUERQUE, V. S. et al. Currículos disciplinares na área da saúde: ensaio sobre saber e poder. Interface - Comunicação, Saúde, Educação, São Paulo, v. 13, n. 31, p. 261-72, out./dez. 2009.

BARDIN, Laurence. Análise de conteúdo. Ed. rev. e ampl. São Paulo: Edições 70, 2011.

BATISTELLA, Carlos. Saúde, doença e cuidado: complexidade teórica e necessidade histórica. In: FONSECA, Angélica Ferreira (Org.). O território e o processo saúde-doença. Rio de Janeiro: EPSJV/Fiocruz, 2007, p. 25-49.

BERBEL, Neusi Aparecida Navas. As metodologias ativas e a promoção da autonomia dos estudantes. Semina: Ciências Sociais e Humanas, Londrina, v. 32, n. 1, p. 25-40, jan./jun. 2011.

BORDAS, Mérion Campos. A interdisciplinaridade na universidade: possibilidades e limites. In: FRANCO, M. E. D. P; KRAHE, E. D. (Orgs.) Pedagogia universitária e áreas do conhecimento. Porto Alegre: EDIPUCRS, 2007, p. 73-93.

BRASIL. Resolução CNE/CES no 3, de 7 de novembro de 2001. Institui as Diretrizes Curriculares Nacionais do Curso de Graduação em Enfermagem. Diário Oficial da União, Brasília, DF, 9 nov. 2001a. Seção 1, p. 37.

BRASIL. Resolução CNE/CES no 5, de 7 de novembro de 2001. Institui as Diretrizes Curriculares Nacionais do Curso de Graduação em Nutrição. Diário Oficial da União, Brasília, DF, 9 nov. 2001b. Seção 1, p. 39. 
BRASIL. Resolução CNE/CES no 2, de 19 de fevereiro de 2002. Institui as Diretrizes Curriculares Nacionais do Curso de Graduação em Farmácia. Diário Oficial da União, Brasília, DF, 4 mar. 2002a. Seção 1, p. 9.

BRASIL. Resolução CNE/CES no 4, de 19 de fevereiro de 2002. Institui as Diretrizes Curriculares Nacionais do Curso de Graduação em Fisioterapia. Diário Oficial da União, Brasília, DF, 4 mar. 2002b. Seção 1, p. 11.

BRASIL. Resolução CNE/CES no 3, de 19 de fevereiro de 2002. Institui as Diretrizes Curriculares Nacionais do Curso de Graduação em Odontologia. Diário Oficial da União, Brasília, DF, 4 mar. 2002c. Seção 1, p. 10.

BRASIL. Ministério da Saúde. Ministério da Educação. A aderência dos cursos de graduação em enfermagem, medicina e odontologia às diretrizes curriculares nacionais. Brasília: Ministério da Saúde, 2006. Disponível em: <http://www.inep.gov.br/pesquisa/ publicacoes> e <http://www.saude.gov.br/sgtes>. Acesso em: 15 abr. 2013.

BRASIL. Ministério da Saúde. Pró-Saúde: objetivos, implementação e desenvolvimento potencial. Brasília: Ministério da Saúde, Ministério da Educação, 2007.

BRASIL. Ministério da Saúde. Relatório do I Seminário Nacional do Programa de Reorientação da Formação Profissional em Saúde II - Pró-Saúde II. Brasília, 2009. 456 p. Disponível em: <www.prosaude.org/publicacoes/>. Acesso em: 15 abr. 2013.

BUSS, Paulo Marchiori. Promoção e educação em saúde no âmbito da Escola de Governo da Escola Nacional de saúde. Cadernos Saúde Pública, Rio de Janeiro, v. 15 (Sup. 2), p. 177-185, 1999. Disponível em: <http://www.scielo.br/pdf/csp/v15s2/1299.pdf>. Acesso em: 20 out. 2013.

DAVINI, Maria Cristina. Currículo Integrado. In: BRASIL. Ministério da Saúde. Capacitação Pedagógica para Instrutor/Supervisor - Área de Saúde. Brasília, 1994. p. 39-48.

FAZENDA, Ivani Catarina Arantes. Integração e interdisciplinaridade no ensino brasileiro: efetividade ou ideologia. 4. ed. São Paulo: Loyola, 1996.

FREIRE, Paulo. Pedagogia do oprimido. 54. ed. Rio de Janeiro: Paz e Terra, 2013.

GATTÁS, Maria Lúcia Borges. Interdisciplinaridade, formação e ação na área da saúde. Ribeirão Preto: Holos, 2006.

GIL, Antônio Carlos. Didática do ensino superior. São Paulo: Atlas, 2011. 
GORDAN, Pedro A. Currículos inovadores: o desafio da inserção docente. In: BATISTA, Nildo A.; BATISTA, Sylvia H. (Orgs.). Docência em saúde: temas e experiências. São Paulo: Editora Senac, 2004. p. 187-200.

LOPES NETO, D. et al. Aderência dos cursos de graduação em Enfermagem às Diretrizes Curriculares Nacionais. Revista Brasileira de Enfermagem, Brasília, v. 60, n. 6, p. 627-634, nov./dez. 2007. Disponível em: <http://www.scielo.br/pdf/reben/v60n6/02.pdf>. Acesso em: 20 jan. 2013.

MACEDO, M. C. S. et al. Cenários de aprendizagem: interseção entre os mundos do trabalho e da formação. In: PINHEIRO, R.; CECCIM, R. B.; MATTOS, R. A. (Orgs.). Ensinar Saúde: a integralidade e o SUS nos cursos de graduação na área da saúde. 2. ed. Rio de Janeiro: CEPESC: IMS/UERJ: ABRASCO, 2011, p. 229-250.

SILVA, E. V. M. et al. A formação de profissionais de saúde em sintonia com o SUS. São Paulo: CONASEMS, 2008, 28 p. Disponível em: <http://www.cosemsms.org.br/ publicacoes/formacao_profissionais_2008.pdf>. Acesso em: 15 set. 2013.

SOUZA, P. A.; ZEFERINO, A. M. B; DA ROS, M. A. Currículo integrado: entre o discurso e a prática. Revista Brasileira de Educação Médica, Rio de Janeiro, v. 35, n. 1, p. 20-25, 2011. Disponível em: <http://www.scielo.br/pdf/rbem/v35n1/a04v35n1.pdf>. Acesso em: 20 ago. 2013.

THIESEN, Juares da Silva. A interdisciplinaridade como um movimento articulador no processo ensino-aprendizagem. Revista Brasileira de Educação [online]. v. 13, n. 39, p. 545-554, 2007. Disponível em: <http://www.scielo.br/scielo.php?script=sci_ pdf\&pid=S141324782008000300010\&lng=en\&nrm=iso\&tlng=pt>. Acesso em: 2 jan. 2013.

TRIVIÑOS. A. N. S. Introdução à pesquisa em ciências sociais: a pesquisa qualitativa em educação. São Paulo: Atlas, 1987.

Universidade Federal dos Vales do Jequitinhonha e Mucuri. Projeto Pedagógico do Curso de Graduação em Enfermagem. Diamantina, MG, 2008a. Disponível em: <http://www. ufvjm.edu.br/prograd/projetos-pedagogicos.html>. Acesso em: 01 jan. 2013.

Universidade Federal dos Vales do Jequitinhonha e Mucuri. Projeto Pedagógico do Curso de Graduação em Farmácia. Diamantina, MG, 2008b. Disponível em: <http://www. ufvjm.edu.br/prograd/projetos-pedagogicos.html>. Acesso em: 01 jan. 2013. 
Universidade Federal dos Vales do Jequitinhonha e Mucuri. Projeto Pedagógico do Curso de Graduação em Fisioterapia. Diamantina, MG, 2009a. Disponível em: <http://www. ufvjm.edu.br/prograd/projetos-pedagogicos.html>. Acesso em: 01 jan. 2013.

Universidade Federal dos Vales do Jequitinhonha e Mucuri. Projeto Pedagógico do Curso de Graduação em Nutrição. Diamantina, MG 2008c. Disponível em: <http://www.ufvjm. edu.br/prograd/projetos-pedagogicos.html>. Acesso em: 01 jan. 2013.

Universidade Federal dos Vales do Jequitinhonha e Mucuri. Projeto Pedagógico do Curso de Graduação em Odontologia. Diamantina, MG, 2009b. Disponível em: <http://www. ufvjm.edu.br/prograd/projetos-pedagogicos.html>. Acesso em: 01 jan. 2013.

VEIGA, IIma P. A. (org.) O projeto político pedagógico da escola: uma construção possível. 11. ed. Campinas: Papirus, 2000.

\section{Lucimar Daniel Simões Salvador}

Mestre em Ensino em Saúde pela Universidade Federal dos Vales do Jequitinhonha e Mucuri (UFVJM) (2014), Diamantina; licenciada em Pedagogia pela Faculdade de Filosofia e Letras de Diamantina (1992); pedagoga da Pró-Reitoria de Graduação da UFVJM.

lucimar.salvador@ufvjm.edu.br

\section{Paulo Afrânio Sant'Anna}

Doutor em Psicologia pela Universidade de São Paulo (2001); docente do curso de licenciatura em Educação do Campo da UFVJM, Diamantina, e do Programa de Pós-Graduação Mestrado Profissional em Ensino em Saúde.

pa.anna1@gmail.com 